\title{
A Finite Element Analysis on Fluid Motion in Librating Triaxial Ellipsoids
}

\author{
Kit H. Chan ${ }^{1}$ Yinnian $\mathrm{He}^{2} \quad$ Keke Zhang $^{3} \quad$ Jun Zou ${ }^{4}$
}

\begin{abstract}
Fluid motion driven by planetary libration may play a key role in maintaining the magnetism of synchronous planets and moons that are thermally or chemically non-convective. We present a fully discrete finite element method on a triaxial ellipsoidal domain for simulating a three-dimensional nonlinear flow in a latitudinally librating triaxial ellipsoidal cavity, for which the usual pseudo-spectral method with the poloidal-toroidal decomposition is difficult because of non-spherical geometry. Stability of the time-dependent finite element solutions with two different temporal schemes are studied, and their error estimates of optimal order are established. The corresponding numerical simulation is implemented for the second-order implicit scheme, offering an insight into the practical aspect of the proposed finite element method.
\end{abstract}

Keywords Finite Element Method, Nonlinear Librating Flow, Triaxial Ellipsoids, Optimal Error estimates

\section{Introduction}

The shape of many planets and moons is, to a first approximation, spherical. It is well known that, however, because of the effect of rapid rotation as well as the interaction among the Sun, planets and moons, many astrophysical bodies are non-spherical and in the shape of a spheroid or a triaxial ellipsoid [18]. As a result of non-spherical geometry, planets and moons are usually rotating non-uniformly and undergo forced libration [20]. It is recently revealed, through an asymptotic analysis [27], that fluid motion in a synchronously rotating spheroidal planet can resonate with planetary latitudinal libration, leading to a large amplitude $\mathrm{O}\left(E^{-1 / 2}\right)$ of the librationally driven flow, where the Ekman number $E$ is extremely small for many rapidly rotating planets. This suggests an alternative driving mechanism for dynamo action within the planets and moons that are thermally or chemically non-convective. A libration-driven dynamo is feasible because the two conditions for resonance - nearly synchronous rotation and small but non-zero eccentricity of the shape - can be approximately met by some synchronous planets and moons.

In comparison with spherical geometry, direct numerical simulation in triaxial ellipsoidal geometry is both mathematically and computationally less tractable. Although triaxial ellipsoidal geometry can be, in principle, accommodated by a coordinate transformation that maps an ellipsoidal domain into the spherical domain [19] or by using complicated non-spherical coordinates [23], there are computational disadvantages in the pseudo-spectral approximation with

\footnotetext{
${ }^{1}$ Department of Mathematics, The University of Hong Kong, Pokfulam, Hong Kong. (mkhchan@hku.hk).

${ }^{2}$ Center for Computational Geosciences, School of Mathematics and Statistics, Xi'an Jiaotong University, China. (heyn@mail.xjtu.edu.cn).

${ }^{3}$ Department of Mathematical Sciences, University of Exeter, EX4 4QF, UK, and the Institute of Mathematical Sciences, Chinese University of Hong Kong, Shatin, Hong Kong. (k.zhang@exeter.ac.uk).

${ }^{4}$ Department of Mathematics, The Chinese University of Hong Kong, Shatin, Hong Kong. (zou@math. cuhk.edu.hk).
} 
the poloidal-toroidal decomposition and, particularly, the mathematical equations resulting from the coordinate transformation are highly complicated. Moreover, the harmonic expansion leads to the global integration that requires an intensive global communication, making it less efficient on modern massively parallel computers. It is thus desirable to seek an alternative numerical method that is non-spectral and can be readily implemented on modern parallel computers for solving the problem of fluid mechanics in librating triaxial ellipsoids.

The present study concerns with the theoretical aspects of finite element methods for simulating the three-dimensional nonlinear flow of a homogeneous fluid of viscosity $\nu$ driven by latitudinal libration and confined within a triaxial ellipsoidal cavity. The triaxial ellipsoidal cavity of arbitrary eccentricity $\mathcal{E}$ is described by

$$
\frac{x^{2}}{a^{2}}+\frac{y^{2}}{a^{2}\left(1+\mathcal{E}^{2}\right)}+\frac{z^{2}}{a^{2}\left(1-\mathcal{E}^{2}\right)}=1,
$$

where $0<\mathcal{E}<1$, which also defines Cartesian coordinates $(x, y, z)$ used in the numerical analysis. The ellipsoidal container rotates rapidly with an angular velocity $\boldsymbol{\Omega}_{0}$ fixed in an inertial frame and, at the same time, undergoes weak latitudinal libration with the libration vector $\boldsymbol{\Omega}_{\text {lat }}$ which results in a periodic variation of the $z$-axis of the ellipsoid towards and away from its mean direction. Through both viscous and topographic coupling between the container and the interior fluid, latitudinal libration can drive fluid motion against viscous dissipation. There are three key parameters that characterize the problem of librationally driven flow in triaxial ellipsoidal cavities: the Ekman number $E=\nu /\left(a^{2} \Omega_{0}\right)$, where $\Omega_{0}=\left|\Omega_{0}\right|$, provides the measure of relative importance between the typical viscous force and the Coriolis force, the eccentricity $\mathcal{E}$ measures the degree of topographic coupling between the container and its interior fluid, and the Poincaré number Po quantifies the strength of Poincaré force resulting from the libration.

For simulating fluid motion driven by latitudinal libration in triaxial ellipsoids, we shall employ an EBE (Element-By-Element) finite element method that has been effectively used for the numerical solution of the dynamo problem in spherical geometry [1]. While the practical aspects of the finite element method, such as how to perform temporal discretization and spatial tetrahedral discretization, have been discussed [2] (see also [3] for the finite element solution of tidally driven flow in a rotating triaxial ellipsoid and [22] for the finite element dynamo), its key theoretical properties, particularly the numerical stability of the finite element scheme and the numerical error of the finite element solution, have not been studied for librationally driven flows in triaxial ellipsoidal geometry. Such theoretical studies will be essential for the geophysical and astrophysical application of the finite element method. The primary purpose of this paper is to understand the theoretical aspects of the finite element method - which is based on the threedimensional triangulation of a triaxial ellipsoidal domain together with the velocity and pressure being represented by continuous piecewise quadratic and linear finite elements - for simulating a nonlinear flow in latitudinally librating triaxial ellipsoids. By providing a mathematical analysis on the numerical stabilities and optimal error estimates of the finite element method, we build a mathematically sound framework that is required for simulating a nonlinear flow in latitudinally librating triaxial ellipsoids.

In what follows we shall begin by presenting the model and governing equations of the numerical problem in Section 2. The theoretical problem of the finite element method is discussed in Section 3 and Section 4. Numerical implementation of the second-order implicit scheme and its results are discussed in Section 5 and the paper closes in Section 6 with a brief summary and some remarks. 


\section{Model and governing equations}

Consider a homogeneous fluid of viscosity $\nu$ confined within a triaxial ellipsoidal cavity defined by (1). Suppose that the ellipsoidal container rotates rapidly with an angular velocity $\Omega_{0}$ which is fixed in the inertial frame and, at the same time, undergoes latitudinal libration with the libration vector $\boldsymbol{\Omega}_{l a t}$ which results in a periodic variation of the $z$-axis slightly towards and away from the rotation axis $\boldsymbol{\Omega}_{0}$. Motivated by its application to synchronous planets and moons, we assume that the overall angular velocity, $\boldsymbol{\Omega}=\boldsymbol{\Omega}_{0}+\boldsymbol{\Omega}_{\text {lat }}$, of the triaxial ellipsoidal container can be expressed as

$$
\boldsymbol{\Omega}=\boldsymbol{\Omega}_{0}+\hat{\mathbf{x}} \Omega_{0} P o \sin \left(\hat{\omega} \Omega_{0} t\right),
$$

where $\hat{\mathbf{x}}$ is a unit vector that is fixed in a frame of reference attached to the container, the mantle frame of reference, and perpendicular to the angular velocity $\boldsymbol{\Omega}_{\mathbf{0}}$, and $\mathrm{Po} / \hat{\boldsymbol{\omega}}$ represents the maximum angular displacement of latitudinal libration with $0<\hat{\omega}<2$. This study is mainly concerned with the key mathematical properties of finite element method for simulating librationally driven flow in a triaxial ellipsoidal cavity.

In the mantle frame of reference, the dynamics of latitudinally librational driven flow is governed by the dimensional equations:

$$
\begin{aligned}
\frac{\partial \mathbf{u}}{\partial t}+\mathbf{u} \cdot & \nabla \mathbf{u}+2 \Omega_{0}\left[\hat{\mathbf{z}}+\hat{\mathbf{x}} P o \sin \left(\Omega_{0} \hat{\omega} t\right)-\hat{\mathbf{y}}(P o / \hat{\omega}) \cos \left(\Omega_{0} \hat{\omega} t\right)\right] \times \mathbf{u}+\frac{1}{\rho} \nabla p \\
= & \nu \nabla^{2} \mathbf{u}+P o \Omega_{0}^{2}\left[\hat{\omega} \mathbf{r} \times \hat{\mathbf{x}} \cos \left(\Omega_{0} \hat{\omega} t\right)+\mathbf{r} \times(\hat{\mathbf{z}} \times \hat{\mathbf{x}}) \sin \left(\Omega_{0} \hat{\omega} t\right)\right], \\
\nabla \cdot \mathbf{u}= & 0,
\end{aligned}
$$

where $\mathbf{r}$ is the position vector, $(\hat{\mathbf{x}}, \hat{\mathbf{y}}, \hat{\mathbf{z}})$ denotes the corresponding unit vectors for the Cartesian coordinates $(x, y, z), p$ is a reduced pressure and $\mathbf{u}$ is the three-dimensional velocity field. The final two terms on the right-hand side of (3) are known as the Poincaré force which results from latitudinal libration and drives fluid motion. Employing the semi-axis $a$ as the length scale, $\Omega_{0}^{-1}$ as the unit of time and $\rho a^{2} \Omega_{0}^{2}$ as the unit of pressure, the non-dimensional envelope of a triaxial ellipsoidal cavity is then described by

$$
\frac{x^{2}}{1}+\frac{y^{2}}{1+\mathcal{E}^{2}}+\frac{z^{2}}{1-\mathcal{E}^{2}}=1
$$

while the non-dimensional governing equations are

$$
\begin{aligned}
\frac{\partial \mathbf{u}}{\partial t}+\mathbf{u} \cdot \nabla \mathbf{u}+2 \hat{\mathbf{z}} \times \mathbf{u}+\nabla p & \\
= & E \nabla^{2} \mathbf{u}+2 \operatorname{Po}[(1 / \hat{\omega}) \hat{\mathbf{y}} \times \mathbf{u} \cos (\hat{\omega} t)-\hat{\mathbf{x}} \times \mathbf{u} \sin (\hat{\omega} t)] \\
& +P o[\hat{\omega} \mathbf{r} \times \hat{\mathbf{x}} \cos (\hat{\omega} t)+\mathbf{r} \times(\hat{\mathbf{z}} \times \hat{\mathbf{x}}) \sin (\hat{\omega} t)], \\
\nabla \cdot \mathbf{u}= & 0 .
\end{aligned}
$$

Note that the centrifugal force is combined with all other conservative forces to form the reduced pressure $p$. Librationally driven flow on the bounding surface, $\mathcal{S}$, of the triaxial ellipsoidal cavity (5) is at rest, requiring that

$$
\hat{\mathbf{n}} \cdot \mathbf{u}=0 ; \quad \hat{\mathbf{n}} \times \mathbf{u}=\mathbf{0}
$$

where $\hat{\mathbf{n}}$ is the normal to $\mathcal{S}$. The problem defined by (6) and (7) subject to the boundary conditions (8) for triaxial ellipsoidal geometry (5) will be solved subject to the initial condition

$$
\mathbf{u}(\mathbf{r}, 0)=\mathbf{u}_{0}(\mathbf{r})
$$


by a three-dimensional fully discrete finite element method.

\section{Finite element method with first-order temporal scheme}

\subsection{Variational formulation and and finite element approximation}

For sake of exposition, we rewrite the governing equations (6)-(7) in the form

$$
\begin{aligned}
\frac{\partial \mathbf{u}}{\partial t}+\mathbf{u} \cdot \nabla \mathbf{u}+\mathbf{Z}(\hat{\omega}, t) \times \mathbf{u}+\nabla p & =E \nabla^{2} \mathbf{u}+\mathbf{f}(\hat{\omega}, x, y, z, t) \text { in } \Omega, \\
\nabla \cdot \mathbf{u} & =0 \text { in } \Omega,
\end{aligned}
$$

where $\Omega$ is the triaxial ellipsoid formed by the interior of the triaxial ellipsoidal cavity $(5), \mathbf{Z}(\hat{\omega}, t)$ and $\mathbf{f}(\hat{\omega}, x, y, z, t)$ are given respectively by

$$
\begin{aligned}
\mathbf{Z}(\hat{\omega}, t) & =2\left[\hat{\mathbf{z}}+P o \hat{\mathbf{x}} \sin (\hat{\omega} t)-P o \hat{\omega}^{-1} \hat{\mathbf{y}} \cos (\hat{\omega} t)\right], \\
\mathbf{f}(\hat{\omega}, x, y, z, t) & =P_{0}[\hat{\omega} \mathbf{r} \times \hat{\mathbf{x}} \cos (\hat{\omega} t)+\mathbf{r} \times(\hat{\mathbf{z}} \times \hat{\mathbf{x}}) \sin (\hat{\omega} t)] .
\end{aligned}
$$

Then we introduce the following trilinear functional

$$
d(\mathbf{w}, \mathbf{u}, \mathbf{v})=\frac{1}{2}\{(\mathbf{w} \cdot \nabla \mathbf{u}, \mathbf{v})-(\mathbf{w} \cdot \nabla \mathbf{v}, \mathbf{u})\} \quad \forall \mathbf{w}, \mathbf{u}, \mathbf{v} \in \mathbf{H}_{0}^{1}(\Omega),
$$

where $(\cdot, \cdot)$ denotes the inner product in $L^{2}(\Omega)^{3}$. Let $L_{0}^{2}(\Omega)$ be the subspace of $L^{2}(\Omega)$ with all functions with a vanishing mean in $\Omega$. Using the trilinear functional (13), we can easily derive the variational formulation to the coupled system (9) and (10) governing the flow $\mathbf{u}$ and the pressure $p$ in the ellipsoid $\Omega$ : Find $\mathbf{u} \in L^{\infty}\left(0, T ; L^{2}(\Omega)^{3}\right) \cap L^{2}\left(0, T ; \mathbf{H}_{0}^{1}(\Omega)\right), p \in L^{2}\left(0, T ; L_{0}^{2}(\Omega)\right)$ such that

$$
\begin{aligned}
\left(\frac{\partial \mathbf{u}}{\partial t}, \mathbf{v}\right)+ & E(\nabla \mathbf{u}, \nabla \mathbf{v})-(p, \nabla \cdot \mathbf{v})+d(\mathbf{u}, \mathbf{u}, \mathbf{v})+(\mathbf{Z} \times \mathbf{u}, \mathbf{v}) \\
& =(\mathbf{f}, \mathbf{v}) \forall \mathbf{v} \in L^{2}\left(0, T ; \mathbf{H}_{0}^{1}(\Omega)\right) \\
-(\nabla \cdot \mathbf{u}, q) & =0 \quad \forall q \in L^{2}\left(0, T ; L_{0}^{2}(\Omega)\right) .
\end{aligned}
$$

Now we are going to propose a fully discrete finite element approximation to the variational system (14) and (15). We start with the partition of the time interval $[0, \mathrm{~T}]$ and the triangulation of the physical ellipsoidal domain $\Omega$. We divide the time interval $[0, \mathrm{~T}]$ into $M$ equally spaced subintervals using the following nodal points

$$
0=t_{0}<t_{1}<t_{2}<\ldots<t_{M}=\mathrm{T},
$$

where $t_{n}=n \tau$ for $n=0,1, \ldots, M$ and $\tau=T / M$. For any given discrete time sequence $\left\{\mathbf{u}^{n}\right\}_{n=0}^{M}$ with each $\mathbf{u}^{n}$ lying in $L^{2}(\Omega)$ or $L^{2}(\Omega)^{3}$, we define the first order backward finite differences and the averages as follows:

$$
\partial_{\tau} \mathbf{u}^{n}=\frac{\mathbf{u}^{n}-\mathbf{u}^{n-1}}{\tau}, \quad \overline{\mathbf{u}}^{n}=\frac{1}{\tau} \int_{t_{n-1}}^{t_{n}} \mathbf{u}(\cdot, s) \mathrm{d} s .
$$

If $\mathbf{u}(\mathbf{r}, t)$ is a function which is continuous with respect to $t$, we shall often write $\mathbf{u}^{n}(\cdot)=\mathbf{u}\left(\cdot, t_{n}\right)$ for $n=0,1, \ldots, M$. 
Next we introduce the triangulation of the ellipsoidal domain $\Omega$. For sake of technical treatments, we shall assume that the domain $\Omega$ is a closed convex polyhedron; the actual ellipsoidal curved boundary case can be treated using some well-developed technicalities for curved boundaries (see., e.g., [4]) in combination with the finite element error estimates established here. Let $\mathcal{T}_{h}$ be a quasi-uniform triangulation of the polyhedral domain $\Omega, \mathbf{V}_{h} \subset \mathbf{H}_{0}^{1}(\Omega)$ and $P_{h} \subset L_{0}^{2}(\Omega)$ be respectively the continuous piecewise quadratic and linear finite element spaces associated with $\mathcal{T}_{h}$. Using the first order semi-implicit scheme, we can now formulate the finite element approximation of the system (14) and (15):

Find $\left\{\mathbf{u}_{h}^{n}\right\} \subset \mathbf{V}_{h}$ and $\left\{p_{h}^{n}\right\} \subset P_{h}$ for $0 \leq n \leq M$ such that $\mathbf{u}_{h}^{0}=I_{h} \mathbf{u}_{0}$ and

$$
\begin{gathered}
\left(\partial_{\tau} \mathbf{u}_{h}^{n}, \mathbf{v}_{h}\right)+E\left(\nabla \mathbf{u}_{h}^{n}, \nabla \mathbf{v}_{h}\right)-\left(p_{h}^{n}, \nabla \cdot \mathbf{v}_{h}\right)+d\left(\mathbf{u}_{h}^{n-1}, \mathbf{u}_{h}^{n}, \mathbf{v}_{h}\right) \\
+\left(\mathbf{Z}^{n} \times \mathbf{u}_{h}^{n}, \mathbf{v}_{h}\right)=\left(\mathbf{f}^{n}, \mathbf{v}_{h}\right) \quad \forall \mathbf{v}_{h} \in \mathbf{V}_{h} \\
-\left(\nabla \cdot \mathbf{u}_{h}^{n}, q_{h}\right)=0 \quad \forall q_{h} \in P_{h} .
\end{gathered}
$$

We end this section with a brief review of some existing results on finite element methods for the standard Navier-Stokes equations and their convergence. For some basic finite element approximations, we refer to the classic monographs $[8,26]$. For the fully discrete finite element approximation of the two-dimensional Navier-Stokes equations, we refer to [10] and [13] for the first order semi-implicit and implicit/explicit temporal schemes and for the stability and convergence under the restrictions $\tau \leq C|\ln h|^{-1}$ and $\tau \leq C$ with the following regularity

(A1) $\mathbf{u} \in L^{\infty}\left(0, T ; \mathbf{H}^{2}(\Omega)\right)$ and $p \in L^{\infty}\left(0, T ; H^{1}(\Omega)\right)$.

But in this work we shall demonstrate the stability and optimal convergence of the finite element solution $\left(\mathbf{u}_{h}^{n}, p_{h}^{n}\right)$ to the first order semi-implicit scheme (16) and (17) in three dimensions, imposing no restrictions on the time step size $\tau$ under the following regularities for the exact solution $(\mathbf{u}, p)$ :

(A2) $\mathbf{u} \in L^{\infty}\left(0, T ; \mathbf{H}^{2}(\Omega)\right) \cap L^{2}\left(0, T ; \mathbf{H}^{3}(\Omega)\right)$ and $\mathbf{u}_{t} \in L^{2}\left(0, T ; \mathbf{H}_{0}^{1}(\Omega)\right), p \in L^{2}\left(0, T ; H^{2}(\Omega)\right)$.

Remark. If domain $\Omega$ is sufficiently smooth, regularities in (A2) can be derived from the Navier-Stokes equations and the regularities in (A1).

\subsection{Auxiliary mathematical and numerical analysis tools}

In this section we shall introduce a few important technical relations, inequalities and finite element interpolation error estimates that are needed for our subsequent convergence analysis for both the first-order temporal scheme in this section and the second-order temporal scheme in next section. Throughout the paper, we shall frequently use $C$ to stand for a generic constant, that is independent of the mesh size $h$, the time stepsize $\tau$ and the relevant functions involved.

By direct computing, one can verify for all $\mathbf{w}, \mathbf{u}, \mathbf{v} \in \mathbf{H}_{0}^{1}(\Omega)$ that

$$
\begin{aligned}
& (\mathbf{Z}(t) \times \mathbf{u}, \mathbf{u})=0, \quad d(\mathbf{w}, \mathbf{u}, \mathbf{u})=0 \\
& d(\mathbf{w}, \mathbf{u}, \mathbf{v})=(\mathbf{w} \cdot \nabla \mathbf{u}, \mathbf{v})+\frac{1}{2}((\nabla \cdot \mathbf{w}) \mathbf{u}, \mathbf{v}),
\end{aligned}
$$

and the following inequalities

$$
\begin{aligned}
& |d(\mathbf{w}, \mathbf{u}, \mathbf{v})| \leq c\|\nabla \mathbf{w}\|_{0}\|\nabla \mathbf{u}\|_{0}\|\nabla \mathbf{v}\|_{0}, \\
& |d(\mathbf{w}, \mathbf{u}, \mathbf{v})| \leq c \sqrt{\|\mathbf{w}\|_{0}\|\nabla \mathbf{w}\|_{0}}\|\nabla \mathbf{u}\|_{0}\|\nabla \mathbf{v}\|_{0} .
\end{aligned}
$$


While for all $\mathbf{w}, \mathbf{v} \in \mathbf{H}_{0}^{1}(\Omega)$ and $\mathbf{u} \in \mathbf{H}^{2}(\Omega) \cap \mathbf{H}_{0}^{1}(\Omega)$ we have

$$
\begin{aligned}
& |d(\mathbf{w}, \mathbf{u}, \mathbf{v})| \leq c\|\mathbf{w}\|_{0}\left(\|\mathbf{u}\|_{L^{\infty}}+\|\nabla \mathbf{u}\|_{L^{3}}\right)\|\nabla \mathbf{v}\|_{0}, \\
& |d(\mathbf{u}, \mathbf{v}, \mathbf{w})| \leq c\left(\|\mathbf{u}\|_{L^{\infty}}+\|\nabla \mathbf{u}\|_{L^{3}}\right)\|\nabla \mathbf{v}\|_{0}\|\mathbf{w}\|_{0} .
\end{aligned}
$$

Here and hereafter, $c$ is used to denote a general positive constant depending only on $\Omega$.

The following two simple relations can be easily verified for any two vector-valued functions $\mathbf{u}, \mathbf{v} \in \mathbf{L}^{2}(\Omega)^{3}$ and any two vectors $\mathbf{a}, \mathbf{b} \in \mathbf{R}^{n}$ :

$$
(\mathbf{u}-\mathbf{v}, \mathbf{u})=\frac{1}{2}\|\mathbf{u}\|_{0}^{2}-\frac{1}{2}\|\mathbf{v}\|_{0}^{2}+\frac{1}{2}\|\mathbf{u}-\mathbf{v}\|_{0}^{2} ; \quad|\mathbf{a} \times \mathbf{b}| \leq \sqrt{2}|\mathbf{a}||\mathbf{b}| .
$$

Let $\mathrm{I}_{h}: L^{2}(\Omega)^{3} \rightarrow \mathbf{V}_{0 h}$ be the standard $L^{2}$-projection. We shall need its following important approximation properties [14]:

$$
\left\|\mathbf{v}-\mathrm{I}_{h} \mathbf{v}\right\|_{0, \Omega}+h\left\|\nabla\left(\mathbf{v}-\mathrm{I}_{h} \mathbf{v}\right)\right\|_{0, \Omega} \leq c h^{i}\|\mathbf{v}\|_{i, \Omega} \quad \forall \mathbf{v} \in \mathbf{H}^{i}(\Omega) \cap \mathbf{V}_{0}
$$

for $i=1,2$, 3, with $\mathbf{V}_{0}=\left\{\mathbf{v} \in \mathbf{H}_{0}^{1}(\Omega) ; \nabla \cdot \mathbf{v}=0\right\}$.

Purely for some subsequent analysis, we shall often make use of the approximate divergencefree finite element space $\mathbf{V}_{0 h}$ :

$$
\mathbf{V}_{0 h}=\left\{\mathbf{v}_{h} \in \mathbf{V}_{h} ; \quad\left(\nabla \cdot \mathbf{v}_{h}, q_{h}\right)=0 \quad \forall q_{h} \in P_{h}\right\} .
$$

We know from [14] that the pair $\left(\mathbf{V}_{h}, P_{h}\right)$ and $\mathbf{V}_{0 h}$ satisfy the following approximation properties:

Property (A). For each $\mathbf{v} \in \mathbf{H}^{i}(\Omega) \cap \mathbf{H}_{0}^{1}(\Omega)$ with $\nabla \cdot \mathbf{v}=0$ and $q \in H^{i-1}(\Omega) \cap L_{0}^{2}(\Omega)$ with $i=1,2,3$, there exist approximations $\pi_{h} \mathbf{v} \in \mathbf{V}_{0 h}$ and $\rho_{h} q \in P_{h}$ such that

$$
\left\|\nabla\left(\mathbf{v}-\pi_{h} \mathbf{v}\right)\right\|_{0} \leq c h^{i-1}\|\mathbf{v}\|_{i}, \quad\left\|q-\rho_{h} q\right\|_{0} \leq c h^{i-1}\|q\|_{i-1} .
$$

As in most finite element analysis, the inverse inequality of the form [5]

$$
\left\|\nabla \mathbf{v}_{h}\right\|_{0} \leq c h^{-1}\left\|\mathbf{v}_{h}\right\|_{0} \quad \forall \mathbf{v}_{h} \in \mathbf{V}_{h}
$$

and the following inf-sup condition [9]: for each $q_{h} \in P_{h}$, there exists $\mathbf{v}_{h} \in \mathbf{V}_{h}, \mathbf{v}_{h} \neq 0$ such that

$$
d\left(\mathbf{v}_{h}, q_{h}\right) \geq \beta\left\|q_{h}\right\|_{0}\left\|\nabla \mathbf{v}_{h}\right\|_{0}
$$

will be very helpful. Here $\beta$ is a positive constant depending only on $\Omega$.

We end this section with the introduction of a discrete Gronwall inequality (see, e.g., [24]): Let $C_{0}, a_{n}, b_{n}, d_{n}$ be nonnegative numbers with integer $n \geq 0$ such that

$$
a_{m}+\tau \sum_{n=1}^{m} b_{n} \leq \tau \sum_{n=0}^{m-1} d_{n} a_{n}+C_{0}, \quad \forall m \geq 1,
$$

then the following estimate holds

$$
a_{m}+\tau \sum_{n=1}^{m} b_{n} \leq C_{0} \exp \left(\tau \sum_{n=0}^{m-1} d_{n}\right), \quad \forall m \geq 1
$$


We emphasize that the Gronwall inequality (25) and (26) is an improved variant of the following one: Let $C_{0}, a_{n}, b_{n}, d_{n}$ be nonnegative numbers with integer $n \geq 0$ such that

$$
a_{m}+\tau \sum_{n=1}^{m} b_{n} \leq \tau \sum_{n=0}^{m} d_{n} a_{n}+C_{0}, \quad \forall m \geq 1 .
$$

If $\tau$ satisfies $\tau d_{n}<1$ for all $0 \leq n \leq m$, then

$$
a_{m}+\tau \sum_{n=1}^{m} b_{n} \leq C_{0} \exp \left(\tau \sum_{n=0}^{m}\left(1-\tau d_{n}\right)^{-1} d_{n}\right), \quad \forall m \geq 1 .
$$

The right-hand side of (27) involves the term $a_{m}$ so the resulting estimate (28) requires a time restriction on $\tau$, while (25) does not. Most existing results, see, e.g., [8] [9] [10] [13] [11] [12] [16] [26], could only reduce their final error estimates to the case with the Gronwall inequality (27) and (28). Instead we shall be able to manipulate the entire error estimate process in a way that our error estimates can finally end with the case for the improved Gronwall inequality (25) and (26). This is one of the key ingredients in our analysis that help us get rid of the time restriction for all our error estimates.

\subsection{Error estimates of finite element solutions}

In this section we establish the stability and error estimates of the discrete solution $\left\{\mathbf{u}_{h}^{n}, p_{h}^{n}\right\}$ to the finite element system (16) and (17).

First for the stability, we take $\mathbf{v}_{h}=\tau \mathbf{u}_{h}^{n}$ in (16) and use (17) to derive

$$
\frac{1}{2}\left\|\mathbf{u}_{h}^{n}\right\|_{0}^{2}-\frac{1}{2}\left\|\mathbf{u}_{h}^{n-1}\right\|_{0}^{2}+\frac{1}{2} \tau^{2}\left\|\partial_{\tau} \mathbf{u}_{h}^{n}\right\|_{0}^{2}+E \tau\left\|\nabla \mathbf{u}_{h}^{n}\right\|_{0}^{2} \leq \tau\left\|\mathbf{f}^{n}\right\|_{0}\left\|\mathbf{u}_{h}^{n}\right\|_{0},
$$

then summing over $n=1,2, \cdots, k \leq M$ and using the Poincaré and Young's inequalities, we obtain the stability estimate:

$$
\begin{aligned}
\max _{1 \leq n \leq M}\left\|\mathbf{u}_{h}^{n}\right\|_{0}^{2} & +\tau \sum_{n=1}^{M}\left(\tau\left\|\partial_{\tau} \mathbf{u}_{h}^{n}\right\|_{0}^{2}+E\left\|\nabla \mathbf{u}_{h}^{n}\right\|_{0}^{2}\right) \\
& \leq c\left(\left\|\mathbf{u}_{h}^{0}\right\|_{0}^{2}+E^{-1} \tau \sum_{n=1}^{M}\left\|\mathbf{f}^{n}\right\|_{0}^{2}\right) .
\end{aligned}
$$

Next, we demonstrate that the finite element solution $\left\{\mathbf{u}_{h}^{n}, p_{h}^{n}\right\}$ to the system (16) and (17) has the optimal error estimates.

Theorem 3.1 Let $(\mathbf{u}, p)$ be the solution to the variational system (14) and (15) with the regularities (A2), and $\left\{\left(\mathbf{u}_{h}^{n}, p_{h}^{n}\right)\right\}$ be the fully discrete solution to the finite element system (16) and (17), then we have the following optimal error estimates

$$
\max _{1 \leq n \leq M}\left\|\mathbf{u}_{h}^{n}-\mathbf{u}^{n}\right\|_{0}^{2}+\tau E \sum_{n=1}^{M}\left\|\nabla\left(\mathbf{u}_{h}^{n}-\mathbf{u}^{n}\right)\right\|_{0}^{2} \leq C\left(\tau^{2}+h^{4}\right) .
$$

Here $C$ is a general positive constant depending on the data $(E, T, \mathbf{u}, \mathbf{Z}, \mathbf{f}, \Omega)$. 
Proof. It suffices to derive the estimate for $\mathbf{u}_{h}^{n}-\mathrm{I}_{h} \mathbf{u}^{n}$ by using the relation

$$
\mathbf{u}_{h}^{n}-\mathbf{u}^{n}=\left(\mathbf{u}_{h}^{n}-\mathrm{I}_{h} \mathbf{u}^{n}\right)+\left(\mathrm{I}_{h} \mathbf{u}^{n}-\mathbf{u}^{n}\right)
$$

and the triangle inequality and the projection approximation (22). So we will estimate $\varepsilon_{h}^{n}:=$ $\mathbf{u}_{h}^{n}-\mathrm{I}_{h} \mathbf{u}^{n}$ below.

Integrating both sides of (14) and (15) over the time interval $\left(t_{n-1}, t_{n}\right)$ respectively, we deduce for any $\mathbf{v} \in \mathbf{H}_{0}^{1}(\Omega)$ and $q \in L^{2}\left(0, T ; L_{0}^{2}(\Omega)\right)$,

$$
\begin{aligned}
\left(\partial_{\tau} \mathbf{u}^{n}, \mathbf{v}\right)+E\left(\nabla \overline{\mathbf{u}}^{n}, \nabla \mathbf{v}\right) & -\left(\bar{p}^{n}, \nabla \cdot \mathbf{v}\right)+\left(\overline{\mathbf{u} \cdot \nabla \mathbf{u}}^{n}, \mathbf{v}\right)+\left(\overline{\mathbf{Z} \times \mathbf{u}}^{n}, \mathbf{v}\right) \\
& =\left(\overline{\mathbf{f}}^{n}, \mathbf{v}\right), \\
-\left(\nabla \cdot \overline{\mathbf{u}}^{n}, q\right) & =0 .
\end{aligned}
$$

Subtracting (32) from (16), we get the following equation for $\varepsilon_{h}^{n}$ :

$$
\begin{aligned}
& \left(\partial_{\tau} \varepsilon_{h}^{n}, \mathbf{v}_{h}\right)+E\left(\nabla \varepsilon_{h}^{n}, \nabla \mathbf{v}_{h}\right)-\left(p_{h}^{n}-\bar{p}^{n}, \nabla \cdot \mathbf{v}_{h}\right) \\
= & \left(\mathbf{f}^{n}-\overline{\mathbf{f}}^{n}, \mathbf{v}_{h}\right)+\left(\overline{\mathbf{u} \cdot \nabla \mathbf{u}}{ }^{n}-\mathbf{u}_{h}^{n-1} \cdot \nabla \mathbf{u}_{h}^{n}, \mathbf{v}_{h}\right)+\left(\overline{\mathbf{Z} \times \mathbf{u}}{ }^{n}-\mathbf{Z}^{n} \times \mathbf{u}_{h}^{n}, \mathbf{v}_{h}\right) \\
+ & E\left(\nabla\left(\overline{\mathbf{u}}^{n}-\mathrm{I}_{h} \mathbf{u}^{n}\right), \nabla \mathbf{v}_{h}\right) .
\end{aligned}
$$

Taking $\mathbf{v}_{h}^{n}=\tau \varepsilon_{h}^{n}$, we obtain

$$
\begin{aligned}
& \frac{1}{2}\left\|\varepsilon_{h}^{n}\right\|_{0}^{2}-\frac{1}{2}\left\|\varepsilon_{h}^{n-1}\right\|_{0}^{2}+\tau E\left\|\nabla \varepsilon_{h}^{n}\right\|_{0}^{2}=\sum_{i=1}^{10}(\mathrm{I})_{i} \\
& \quad \equiv \int_{t_{n-1}}^{t_{n}}\left(t-t_{n-1}\right)\left(\mathbf{f}_{t}(t), \varepsilon_{h}^{n}\right) d t-\int_{t_{n-1}}^{t_{n}}\left(t-t_{n-1}\right) d_{t}\left(\mathbf{u}, \mathbf{u}, \varepsilon_{h}^{n}\right) d t \\
& \quad+\tau d\left(\mathbf{u}\left(t_{n}\right)-\mathbf{u}\left(t_{n-1}\right), \mathbf{u}\left(t_{n}\right), \varepsilon_{h}^{n}\right)+d\left(\mathbf{u}\left(t_{n-1}\right)-I_{h} \mathbf{u}\left(t_{n-1}\right)+\varepsilon_{h}^{n-1}, \mathbf{u}\left(t_{n}\right), \varepsilon_{h}^{n}\right) \\
& \quad+\tau d\left(\mathbf{u}_{h}^{n-1}, \mathbf{u}\left(t_{n}\right)-I_{h} \mathbf{u}\left(t_{n}\right), \varepsilon_{h}^{n}\right)+\tau\left(\rho_{h} \bar{p}^{n}-\bar{p}^{n}, \nabla \cdot \varepsilon_{h}^{n}\right) \\
& \quad+\quad \int_{t_{n-1}}^{t_{n}}\left(t-t_{n-1}\right)\left(\mathbf{Z}_{t} \times \mathbf{u}+\mathbf{Z} \times \mathbf{u}_{t}, \varepsilon_{h}^{n}\right) d t+\left(\mathbf{Z}\left(t_{n}\right) \times\left(\mathbf{u}\left(t_{n}\right)-I_{h} \mathbf{u}\left(t_{n}\right)\right), \varepsilon_{h}^{n}\right) \\
& \quad-\quad E \int_{t_{n-1}}^{t_{n}}\left(t-t_{n-1}\right)\left(\nabla \mathbf{u}_{t}, \nabla \varepsilon_{h}^{n}\right) d t+E \tau\left(\nabla\left(\mathbf{u}\left(t_{n}\right)-I_{h} \mathbf{u}\left(t_{n}\right)\right), \nabla \varepsilon_{h}^{n}\right) .
\end{aligned}
$$

Now by some standard techniques and using the Poincaré and Young inequalities, we can derive the following estimates for all the terms $(\mathrm{I})_{1}$ to $(\mathrm{I})_{10}$ in (34) except for $(\mathrm{I})_{2}$ and $(\mathrm{I})_{3}$ :

$$
\begin{aligned}
(\mathrm{I})_{1} & \leq c \tau^{\frac{3}{2}}\left(\int_{t_{n-1}}^{t_{n}}\left\|\mathbf{f}_{t}\right\|_{0}^{2} d t\right)^{\frac{1}{2}}\left\|\varepsilon_{h}^{n}\right\|_{0} \leq \frac{E}{16}\left\|\nabla \varepsilon_{h}^{n}\right\|_{0}^{2} \tau+c E^{-1} \tau^{2} \int_{t_{n-1}}^{t_{n}}\left\|\mathbf{f}_{t}\right\|_{0}^{2} d t, \\
(\mathrm{I})_{4} & \leq c \tau\left\|\varepsilon_{h}^{n-1}\right\|_{0}\left\|\mathbf{u}\left(t_{n}\right)\right\|_{2}\left\|\nabla \varepsilon_{h}^{n}\right\|_{0} \\
& +c \tau\left\|\nabla\left(\mathbf{u}\left(t_{n-1}\right)-I_{h} \mathbf{u}\left(t_{n-1}\right)\right)\right\|_{0}\left\|\nabla \mathbf{u}\left(t_{n}\right)\right\|_{0}\left\|\nabla \varepsilon_{h}^{n}\right\|_{0} \\
& \leq \frac{E}{16}\left\|\nabla \varepsilon_{h}^{n}\right\|_{0}^{2} \tau+c E^{-1} \tau\left\|\nabla\left(\mathbf{u}\left(t_{n-1}\right)-I_{h} \mathbf{u}\left(t_{n-1}\right)\right)\right\|_{0}^{2}\left\|\nabla \mathbf{u}\left(t_{n}\right)\right\|_{0}^{2} \\
& +c E^{-1} \tau\left\|\varepsilon_{h}^{n-1}\right\|_{0}^{2}\left\|\mathbf{u}\left(t_{n}\right)\right\|_{2}^{2}
\end{aligned}
$$




$$
\begin{aligned}
(\mathrm{I})_{5} & \leq c h^{-1} \tau\left\|\varepsilon_{h}^{n-1}\right\|_{0}\left\|\nabla\left(\mathbf{u}\left(t_{n}\right)-I_{h} \mathbf{u}_{(}\left(t_{n}\right)\right)\right\|_{0}\left\|\nabla \varepsilon_{h}^{n}\right\|_{0} \\
& +c \tau \| \nabla\left(I_{h} \mathbf{u}\left(t_{n-1}\right)\left\|_{0}\right\| \nabla\left(\mathbf{u}\left(t_{n}\right)-I_{h} \mathbf{u}\left(t_{n}\right)\right)\left\|_{0}\right\| \nabla \varepsilon_{h}^{n} \|_{0}\right. \\
& \leq \frac{E}{16}\left\|\nabla \varepsilon_{h}^{n}\right\|_{0}^{2} \tau+c E^{-1} \tau\left\|\nabla \mathbf{u}\left(t_{n-1}\right)\right\|_{0}\left\|\nabla\left(\mathbf{u}\left(t_{n}\right)-I_{h} \mathbf{u}\left(t_{n}\right)\right)\right\|_{0}^{2} \\
& +c E^{-1} \tau\left\|\varepsilon_{h}^{n-1}\right\|_{0}^{2}\left\|\mathbf{u}\left(t_{n}\right)\right\|_{2}^{2} \\
(\mathrm{I})_{6} & \leq c \tau\left\|\bar{p}^{n}-\rho_{h} \bar{p}^{n}\right\|_{0}\left\|\nabla \varepsilon_{h}^{n}\right\|_{0} \leq \frac{E}{16}\left\|\nabla \varepsilon_{h}^{n}\right\|_{0}^{2} \tau+c E^{-1} h^{4} \int_{t_{n-1}}^{t_{n}}\|p\|_{2}^{2} d t \\
(\mathrm{I})_{7} & \leq c \tau^{\frac{3}{2}}\left(\int_{t_{n-1}}^{t_{n}}\left(\left|\mathbf{Z}_{t}\right|^{2}\|\mathbf{u}\|_{0}^{2}+|\mathbf{Z}|^{2}\left\|\mathbf{u}_{t}\right\|_{0}^{2}\right) d t\right)^{\frac{1}{2}}\left\|\nabla \varepsilon_{h}^{n}\right\|_{0} \\
& \leq \frac{E}{16}\left\|\nabla \varepsilon_{h}^{n}\right\|_{0}^{2} \tau+c E^{-1} \tau^{2} \int_{t_{n-1}}^{t_{n}}\left(\left|\mathbf{Z}_{t}\right|^{2}\|\mathbf{u}\|_{0}^{2}+|\mathbf{Z}|^{2}\left\|\mathbf{u}_{t}\right\|_{0}^{2}\right) d t \\
(\mathrm{I})_{8} & \leq c \tau\left|\mathbf{Z}\left(t_{n}\right)\right|\left\|\mathbf{u}\left(t_{n}\right)-I_{h} \mathbf{u}\left(t_{n}\right)\right\|_{0}\left\|\varepsilon_{h}^{n}\right\|_{0} \\
& \leq \frac{E}{16}\left\|\nabla \varepsilon_{h}^{n}\right\|_{0}^{2} \tau+c E^{-1} \tau\left|\mathbf{Z}\left(t_{n}\right)\right|^{2}\left\|\nabla\left(\mathbf{u}\left(t_{n}\right)-I_{h} \mathbf{u}\left(t_{n}\right)\right)\right\|_{0}^{2}, \\
(\mathrm{I})_{9} & \leq c \tau^{\frac{3}{2}}\left(\int_{t_{n-1}}^{t_{n}}\left\|\nabla \mathbf{u}_{t}\right\|_{0}^{2} d t\right)^{\frac{1}{2}}\left\|\nabla \varepsilon_{h}^{n}\right\|_{0} \leq \frac{E}{16}\left\|\nabla \varepsilon_{h}^{n}\right\|_{0}^{2} \tau+c E^{-1} \tau^{2} \int_{t_{n-1}}^{t_{n}}\left\|\nabla \mathbf{u}_{t}\right\|_{0}^{2} d t \\
(\mathrm{I})_{10} & \leq c \tau\left\|\nabla\left(\mathbf{u}\left(t_{n}\right)-I_{h} \mathbf{u}\left(t_{n}\right)\right)\right\|_{0}\left\|\nabla \varepsilon_{h}^{n}\right\|_{0} \\
& \leq \frac{E}{16}\left\|\nabla \varepsilon_{h}^{n}\right\|_{0}^{2} \tau+c E^{-1} \tau\left\|\nabla\left(\mathbf{u}\left(t_{n}\right)-\mathbf{u}\left(t_{n}\right)\right)\right\|_{0}^{2} .
\end{aligned}
$$

On the other hand, by using (21) and the following inequality

$$
\|\mathbf{u}\|_{L^{\infty}}+\|\nabla \mathbf{u}\|_{L^{3}} \leq c\|\mathbf{u}\|_{2} \quad \forall \mathbf{u} \in \mathbf{H}^{2}(\Omega) \cap \mathbf{H}_{0}^{1}(\Omega),
$$

we can estimate $(\mathrm{I})_{2}$ and $(\mathrm{I})_{3}$ as follows:

$$
\begin{aligned}
(\mathrm{I})_{2} & \leq c \int_{t_{n-1}}^{t_{n}}\left(t-t_{n-1}\right)\left[\left|d\left(\mathbf{u}_{t}, \mathbf{u}, \varepsilon_{h}^{n}\right)\right|+\left|d\left(\mathbf{u}, \varepsilon_{h}^{n}, \mathbf{u}_{t}\right)\right|\right] d t \\
& \leq c \tau^{\frac{3}{2}}\left(\int_{t_{n-1}}^{t_{n}}\left\|\mathbf{u}_{t}\right\|_{0}^{2}\|\mathbf{u}\|_{2}^{2} d t\right)^{\frac{1}{2}}\left\|\nabla \varepsilon_{h}^{n}\right\|_{0} \\
& \leq \frac{E}{16}\left\|\nabla \varepsilon_{h}^{n}\right\|_{0}^{2} \tau+c E^{-1} \tau^{2} \int_{t_{n-1}}^{t_{n}}\left\|\mathbf{u}_{t}\right\|_{0}^{2}\|\mathbf{u}\|_{2}^{2} d t \\
(\mathrm{I})_{3} & \leq c \tau^{\frac{3}{2}}\left(\int_{t_{n-1}}^{t_{n}}\left\|\mathbf{u}_{t}\right\|_{0}^{2} d t\right)^{\frac{1}{2}}\left\|\mathbf{u}\left(t_{n}\right)\right\|_{2}\left\|\nabla \varepsilon_{h}^{n}\right\|_{0} \\
& \leq \frac{E}{16}\left\|\nabla \varepsilon_{h}^{n}\right\|_{0}^{2} \tau+c E^{-1} \tau^{2} \int_{t_{n-1}}^{t_{n}}\left\|\mathbf{u}_{t}\right\|_{0}^{2}\left\|\mathbf{u}\left(t_{n}\right)\right\|_{2}^{2} d t
\end{aligned}
$$

Summing up (34) over $n=1$ to $n=m$ and using the above estimates for $(\mathrm{I})_{1}$ to $(\mathrm{I})_{10}$ and the regularities (A2), lead us to the following bound:

$$
\begin{aligned}
& \left\|\varepsilon_{h}^{m}\right\|_{0}^{2}+E \tau \sum_{n=1}^{m}\left\|\nabla \varepsilon_{h}^{n}\right\|_{0}^{2} \leq C\left(\tau^{2}+h^{4}\right) \int_{0}^{T}\left(\left\|\mathbf{f}_{t}\right\|_{0}^{2}+\|\mathbf{u}\|_{0}^{2}+\left\|\mathbf{u}_{t}\right\|_{1}^{2}+\|p\|_{2}^{2}\right) d t \\
& \quad+C \tau \sum_{n=1}^{M}\left\|\nabla\left(\mathbf{u}\left(t_{n}\right)-I_{h} \mathbf{u}\left(t_{n}\right)\right)\right\|_{0}^{2}+c E^{-1} \tau \sum_{n=1}^{m-1}\left\|\mathbf{u}\left(t_{n+1}\right)\right\|_{2}^{2}\left\|\varepsilon_{h}^{n}\right\|_{0}^{2} .
\end{aligned}
$$


Then applying the discrete Gronwall inequality to (36) and using (A2), we further deduce

$$
\begin{aligned}
& \left\|\varepsilon_{h}^{m}\right\|_{0}^{2}+E \tau \sum_{n=1}^{m}\left\|\nabla \varepsilon_{h}^{n}\right\|_{0}^{2} \\
\leq & C \exp \left\{c E^{-1} \tau \sum_{n=1}^{m-1}\left\|\mathbf{u}\left(t_{n+1}\right)\right\|_{2}^{2}\right\}\left\{\tau^{2}+h^{4}+\tau \sum_{n=1}^{M}\left\|\nabla\left(\mathbf{u}\left(t_{n}\right)-I_{h} \mathbf{u}\left(t_{n}\right)\right)\right\|_{0}^{2}\right\} .
\end{aligned}
$$

But it follows easily that

$$
\tau\left\|\nabla\left(\mathbf{u}\left(t_{n}\right)-I_{h} \mathbf{u}\left(t_{n}\right)\right)\right\|_{0}^{2} \leq c\left(\tau^{2}+h^{4}\right) \int_{t_{n-1}}^{t_{n}}\left[\left\|\nabla \mathbf{u}_{t}\right\|_{0}^{2}+\|\mathbf{u}\|_{3}^{2}\right] d t
$$

by writing $\mathbf{u}\left(t_{n}\right)-I_{h} \mathbf{u}\left(t_{n}\right)=\left(\mathbf{u}\left(t_{n}\right)-\overline{\mathbf{u}}^{n}\right)+\left(\overline{\mathbf{u}}^{n}-I_{h} \overline{\mathbf{u}}^{n}\right)+\left(I_{h} \overline{\mathbf{u}}^{n}-I_{h} \mathbf{u}\left(t_{n}\right)\right)$. Now the desired estimate (30) follows from (31) by the triangle inequality, (37) and (38), and the projection approximation (22). $\sharp$

\section{Finite element method with second-order temporal scheme}

In the previous section we have discussed a fully discrete finite element method with first-order time marching scheme. But for our highly nonlinear libration system, the first-order scheme may not be always sufficient to capture the accuracy of the flow in an effective and stable manner. In this section we present a more accurate time discretization, the second order Crank-Nicolson extrapolation scheme. The subsequent notations for the time and space discretizations as well as the finite element spaces are all carried over from the previous section.

Now we are going to use the implicit second order Crank-Nicolson scheme for the linear terms and the implicit second order extrapolation to deal with the nonlinear term. We shall also write

$$
\overline{\mathbf{u}}^{n+1 / 2}=\frac{1}{\tau} \int_{t_{n}}^{t_{n+1}} \mathbf{u}(s) \mathrm{d} s, \quad \mathbf{u}^{n+1 / 2}=\frac{\mathbf{u}^{n+1}+\mathbf{u}^{n}}{2}, \quad \mathbf{u}_{h}^{n+1 / 2}=\frac{\mathbf{u}_{h}^{n+1}+\mathbf{u}_{h}^{n}}{2}
$$

and

$$
T_{n}(\mathbf{u})=\frac{3}{2} \mathbf{u}^{n}-\frac{1}{2} \mathbf{u}^{n-1} \quad \text { or } \quad T_{n}\left(\mathbf{u}_{h}\right)=\frac{3}{2} \mathbf{u}_{h}^{n}-\frac{1}{2} \mathbf{u}_{h}^{n-1} .
$$

Using these approximations in time along with the same finite element approximations as used in the previous section in space, we propose the following fully discrete finite element scheme for the system (14) and (15):

Find $\left\{\mathbf{u}_{h}^{n}\right\} \subset \mathbf{V}_{h},\left\{p_{h}^{n}\right\} \subset P_{h}$ for $n=0,1, \cdots, M$ such that $\mathbf{u}_{h}^{0}=\mathrm{I}_{h} \mathbf{u}_{0}$ and

$$
\begin{aligned}
& \left(\partial_{\tau} \mathbf{u}_{h}^{n+1}, \mathbf{v}_{h}\right)+E\left(\nabla \mathbf{u}_{h}^{n+1 / 2}, \nabla \mathbf{v}_{h}\right)-\left(p_{h}^{n+1 / 2}, \nabla \cdot \mathbf{v}_{h}\right) \\
& +d\left(T_{n}\left(\mathbf{u}_{h}\right), \mathbf{u}_{h}^{n+1 / 2}, \mathbf{v}_{h}\right)+\left(\mathbf{Z}^{n+1 / 2} \times \mathbf{u}_{h}^{n+1 / 2}, \mathbf{v}_{h}\right)=\left(\mathbf{f}^{n+1 / 2}, \mathbf{v}_{h}\right) \quad \forall \mathbf{v}_{h} \in \mathbf{V}_{h} \\
& -\left(\nabla \cdot \mathbf{u}_{h}^{n+1 / 2}, q_{h}\right)=0 \quad \forall q_{h} \in P_{h} .
\end{aligned}
$$

It is easy to see that the scheme (39) and (40) just needs to solve a linear system at each time step, and it will be shown to be second accurate in time. 
It is well-known [16] that for the fully implicit second order Crank-Nicolson scheme based on the mixed finite element method, there is a restriction $\tau \leq C$ on the time step size for the convergence. While for the semi-implicit second order Crank-Nicolson extrapolation scheme, there is also a restriction $\tau \leq C$ on the time step size; see [11] for the 2D Navier-Stokes equations. On the other hand, for the semi-implicit second order Crank-Nicolson extrapolation scheme based on the stabilized finite element method, no restrictions are imposed on the time step size but the convergence rate of the scheme is only of order $O\left(\tau^{\frac{3}{2}}\right)$ in time; see [12] for the 2D Navier-Stokes equations.

To the best of our knowledge, this seems to be the first time to establish the optimal second order convergence of the discrete solution $\left(\mathbf{u}_{h}^{n}, p_{h}^{n}\right)$ to a linearized finite element system of the Navier-Stokes equations in three dimensions, and more importantly, the optimal convergence rate will be achieved without imposing any restriction on the time step size $\tau$, under the following reasonable assumptions on the regularities of the exact solution $(\mathbf{u}, p)$ :

$$
\begin{cases}\mathbf{u} \in L^{\infty}\left(0, T ; \mathbf{H}^{2}(\Omega)\right) \cap L^{2}\left(0, T ; \mathbf{H}^{3}(\Omega)\right), & p \in L^{2}\left(0, T ; H^{2}(\Omega)\right), \\ \mathbf{u}_{t} \in L^{\infty}\left(0, T ; \mathbf{H}^{1}(\Omega)\right) \cap L^{2}\left(0, T ; \mathbf{H}^{2}(\Omega)\right), & \mathbf{u}_{t t} \in L^{2}\left(0, T ; \mathbf{H}^{1}(\Omega)\right) .\end{cases}
$$

\subsection{Error estimates of finite element solutions}

In this section we establish the error estimate of the discrete solutions $\left\{\mathbf{u}_{h}^{n}, p_{h}^{n}\right\}$ to the finite element system (39) and (40). For this, we first derive the stability estimates for $\left\{\mathbf{u}_{h}^{n}, p_{h}^{n}\right\}$. By choosing $\mathbf{v}_{h}=\tau \mathbf{u}_{h}^{n+1 / 2}$ in (39) we obtain

$$
\frac{1}{2}\left\|\mathbf{u}_{h}^{n+1}\right\|_{0}^{2}-\frac{1}{2}\left\|\mathbf{u}_{h}^{n}\right\|_{0}^{2}+E \tau\left\|\nabla \mathbf{u}_{h}^{n+1 / 2}\right\|_{0}^{2} \leq \frac{E}{2}\left\|\nabla \mathbf{u}_{h}^{n}\right\|_{0}^{2}+C \tau\left\|\mathbf{f}^{n+1 / 2}\right\|_{0}^{2},
$$

then summing over $n=1,2, \cdots, k \leq M$, we come to the stability estimate:

$$
\max _{1 \leq n \leq M}\left\|\mathbf{u}_{h}^{n}\right\|_{0}^{2}+E \sum_{n=1}^{M} \tau\left\|\nabla \mathbf{u}_{h}^{n+1 / 2}\right\|_{0}^{2} \leq C\left(\left\|\mathbf{u}_{h}^{0}\right\|_{0}^{2}+\sum_{n=1}^{M} \tau\left\|\mathbf{f}^{n}\right\|_{0}^{2}\right) .
$$

Now we are ready to demonstrate the optimal error estimate of the finite element solutions $\left\{\mathbf{u}_{h}^{n}, p_{h}^{n}\right\}$ to the system (39) and (40), second order accurate in both space and time.

Theorem 4.1 Let $(\mathbf{u}, p)$ be the solution to the variational system (14) and (15) with the regularities (A3), and $\left\{\left(\mathbf{u}_{h}^{n}, p_{h}^{n}\right)\right\}$ be the fully approximate solution to the finite element system (39) and (40). Then we have the following optimal error estimates

$$
\max _{1 \leq n \leq M}\left\|\mathbf{u}_{h}^{n}-\mathbf{u}^{n}\right\|_{0}^{2}+\tau E \sum_{n=1}^{M}\left\|\nabla\left(\mathbf{u}_{h}^{n}-\mathbf{u}^{n}\right)\right\|_{0}^{2} \leq C\left(\tau^{4}+h^{4}\right) .
$$

Proof. Similarly as we argued in the proof of Theorem 3.1, it suffices to estimate the error $\varepsilon_{h}^{n}=\mathbf{u}_{h}^{n}-\mathrm{I}_{h} \mathbf{u}^{n}$. To derive the equation satisfied by $\varepsilon_{h}^{n}$, we integrate both sides of (14) and (15) over the time interval $\left(t_{n}, t_{n+1}\right)$ respectively to deduce for any $\mathbf{v} \in \mathbf{H}_{0}^{1}(\Omega)$ and $q \in L_{0}^{2}(\Omega)$,

$$
\begin{aligned}
\left(\partial_{\tau} \mathbf{u}^{n+1}, \mathbf{v}\right)+E\left(\nabla \overline{\mathbf{u}}^{n+1 / 2}, \nabla \mathbf{v}\right)-\left(\bar{p}^{n+1 / 2}, \nabla \cdot \mathbf{v}\right)+\left(\overline{\mathbf{u} \cdot \nabla \mathbf{u}}^{n+1 / 2}, \mathbf{v}\right) \\
+\quad\left(\overline{\mathbf{Z} \times \mathbf{u}}{ }^{n+1 / 2}, \mathbf{v}\right)=\left(\overline{\mathbf{f}}^{n+1 / 2}, \mathbf{v}\right), \\
-\left(\nabla \cdot \overline{\mathbf{u}}^{n+1 / 2}, q\right)=0 .
\end{aligned}
$$


Subtracting (43) from (39) yields the equation for the error function $\varepsilon_{h}^{n}$ :

$$
\begin{aligned}
& \left(\partial_{\tau} \varepsilon_{h}^{n+1}, \mathbf{v}_{h}\right)+E\left(\nabla \varepsilon_{h}^{n+1 / 2}, \nabla \mathbf{v}_{h}\right)-\left(p_{h}^{n+1 / 2}-\bar{p}^{n+1 / 2}, \nabla \cdot \mathbf{v}_{h}\right) \\
= & \left(\mathbf{f}^{n+1 / 2}-\overline{\mathbf{f}}^{n+1 / 2}, \mathbf{v}_{h}\right)+\left(\overline{\mathbf{u} \cdot \nabla \mathbf{u}}{ }^{n+1 / 2}-T_{n}\left(\mathbf{u}_{h}\right) \cdot \nabla \mathbf{u}_{h}^{n+1 / 2}, \mathbf{v}_{h}\right) \\
& +\left(\overline{\mathbf{Z} \times \mathbf{u}}{ }^{n+1 / 2}-\mathbf{Z}^{n+1 / 2} \times \mathbf{u}_{h}^{n+1 / 2}, \mathbf{v}_{h}\right)+\left(\partial_{\tau}\left(\mathbf{u}^{n+1}-\mathrm{I}_{h} \mathbf{u}^{n+1}\right), \mathbf{v}_{h}\right) \\
& +E\left(\nabla\left(\overline{\mathbf{u}}^{n+1 / 2}-\mathrm{I}_{h} \mathbf{u}^{n+1 / 2}\right), \nabla \mathbf{v}_{h}\right) .
\end{aligned}
$$

Taking $\mathbf{v}_{h}^{n}=\tau \varepsilon_{h}^{n+1 / 2}$ in the above equation, we can rewrite it as follows:

$$
\begin{aligned}
& \frac{1}{2}\left\|\varepsilon_{h}^{n+1}\right\|_{0}^{2}-\frac{1}{2}\left\|\varepsilon_{h}^{n}\right\|_{0}^{2}+\tau E\left\|\nabla \varepsilon_{h}^{n+1 / 2}\right\|_{0}^{2} \equiv \sum_{i=1}^{12}(\mathrm{I})_{i} \\
& =\frac{1}{2} \int_{t_{n}}^{t_{n+1}}\left(t-t_{n}\right)\left(t_{n+1}-t\right)\left(\mathbf{f}_{t t}, \varepsilon_{h}^{n+1 / 2}\right) d t \\
& -\frac{1}{2} \int_{t_{n}}^{t_{n+1}}\left(t-t_{n}\right)\left(t_{n+1}-t\right)\left(\mathbf{Z}_{t t} \times \mathbf{u}+2 \mathbf{Z}_{t} \times \mathbf{u}_{t}+\mathbf{Z} \times \mathbf{u}_{t t}, \varepsilon_{h}^{n+\frac{1}{2}}\right) \\
& +\frac{\tau}{4}\left(\left(\mathbf{Z}\left(t_{n+1}\right)-\mathbf{Z}\left(t_{n}\right)\right) \times\left(\mathbf{u}\left(t_{n+1}\right)-\mathbf{u}\left(t_{n}\right)\right), \varepsilon_{h}^{n+\frac{1}{2}}\right) \\
& +\tau\left(\mathbf{Z}^{n+\frac{1}{2}} \times\left(\mathbf{u}^{n+\frac{1}{2}}-I_{h} \mathbf{u}^{n+\frac{1}{2}}\right), \varepsilon_{h}^{n+\frac{1}{2}}\right) \\
& -\frac{E}{2} \int_{t_{n}}^{t_{n+1}}\left(t-t_{n}\right)\left(t_{n+1}-t\right)\left(\nabla \mathbf{u}_{t t}, \nabla \varepsilon_{h}^{n+\frac{1}{2}}\right) d t+E \tau\left(\nabla\left(\mathbf{u}^{n+\frac{1}{2}}-I_{h} \mathbf{u}^{n+\frac{1}{2}}\right), \nabla \varepsilon_{h}^{n}\right) \\
& +\tau\left(\rho_{h} \bar{p}^{n+\frac{1}{2}}-\bar{p}^{n+\frac{1}{2}}, \nabla \cdot \varepsilon_{h}^{n+\frac{1}{2}}\right)-\frac{1}{2} \int_{t_{n}}^{t_{n+1}}\left(t-t_{n}\right)\left(t_{n+1}-t\right) d_{t t}\left(\mathbf{u}, \mathbf{u}, \varepsilon_{h}^{n+\frac{1}{2}}\right) d t \\
& +\frac{\tau}{4} d\left(\mathbf{u}\left(t_{n+1}\right)-\mathbf{u}\left(t_{n}\right), \mathbf{u}\left(t_{n+1}\right)-\mathbf{u}\left(t_{n}\right), \varepsilon_{h}^{n}\right) \\
& +\frac{\tau}{2} d\left(\mathbf{u}\left(t_{n+1}-2 \mathbf{u}\left(t_{n}\right)+\mathbf{u}\left(t_{n-1}\right), \mathbf{u}^{n+\frac{1}{2}}, \varepsilon_{h}^{n+\frac{1}{2}}\right)\right. \\
& +\tau d\left(T_{n}(\mathbf{u})-I_{h} T_{n}(\mathbf{u})+T_{n}\left(\varepsilon_{h}\right), \mathbf{u}^{n+\frac{1}{2}}, \varepsilon_{h}^{n+\frac{1}{2}}\right)+\tau d\left(T_{n}\left(\mathbf{u}_{h}\right), \mathbf{u}^{n+\frac{1}{2}}-I_{h} \mathbf{u}^{n+\frac{1}{2}}, \varepsilon_{h}^{n+\frac{1}{2}}\right) .
\end{aligned}
$$


By some standard techniques, we can estimate $(\mathrm{I})_{1}$ to $(\mathrm{I})_{7}$ as

$$
\begin{aligned}
(\mathrm{I})_{1} & \leq c \tau^{\frac{5}{2}}\left(\int_{t_{n}}^{t_{n+1}}\left\|\mathbf{f}_{t t}\right\|_{0}^{2} d t\right)^{\frac{1}{2}}\left\|\varepsilon_{h}^{n+\frac{1}{2}}\right\|_{0} \leq \frac{E \tau}{16}\left\|\nabla \varepsilon_{h}^{n+\frac{1}{2}}\right\|_{0}^{2}+\frac{c \tau^{4}}{E} \int_{t_{n}}^{t_{n+1}}\left\|\mathbf{f}_{t t}\right\|_{0}^{2} d t, \\
(\mathrm{I})_{2} & \leq c \tau^{\frac{5}{2}}\left(\int_{t_{n}}^{t_{n+1}}\left[\left|\mathbf{Z}_{t t}\right|^{2}\|\mathbf{u}\|_{0}^{2}+\left|\mathbf{Z}_{t}\right|^{2}\left\|\mathbf{u}_{t}\right\|_{0}^{2}+|\mathbf{Z}|^{2}\left\|\mathbf{u}_{t t}\right\|_{0}^{2}\right] d t\right)^{\frac{1}{2}}\left\|\varepsilon_{h}^{n+\frac{1}{2}}\right\|_{0} \\
& \leq \frac{E}{16}\left\|\nabla \varepsilon_{h}^{n+\frac{1}{2}}\right\|_{0}^{2} \tau+c E^{-1} \tau^{4} \int_{t_{n-1}}^{t_{n}}\left[\|\mathbf{u}\|_{0}^{2}+\left\|\mathbf{u}_{t}\right\|_{0}^{2}+\left\|\mathbf{u}_{t t}\right\|_{0}^{2}\right] d t \\
(\mathrm{I})_{3} & \leq c \tau \int_{t_{n}}^{t_{n+1}}\left|\mathbf{Z}_{t}\right| d t \int_{t_{n}}^{t_{n+1}}\left\|\mathbf{u}_{t}\right\|_{0} d t\left\|\varepsilon_{h}^{n+\frac{1}{2}}\right\|_{0} \\
& \leq \frac{E}{16}\left\|\nabla \varepsilon_{h}^{n+\frac{1}{2}}\right\|_{0}^{2} \tau+c E^{-1} \tau^{4} \int_{t_{n}}^{t_{n+1}}\left\|\mathbf{u}_{t}\right\|_{0}^{2} d t \\
(\mathrm{I})_{4} & \leq c \tau\left|\mathbf{Z}^{n+\frac{1}{2}}\right|\left\|\mathbf{u}^{n+\frac{1}{2}}-I_{h} \mathbf{u}^{n+\frac{1}{2}}\right\|_{0}\left\|\varepsilon_{h}^{n+\frac{1}{2}}\right\|_{0} \\
& \leq \frac{E}{16}\left\|\nabla \varepsilon_{h}^{n+\frac{1}{2}}\right\|_{0}^{2} \tau+c E^{-1} \tau\left\|\nabla\left(\mathbf{u}^{n+\frac{1}{2}}-I_{h} \mathbf{u}^{n+\frac{1}{2}}\right)\right\|_{0}^{2}, \\
(\mathrm{I})_{5} & \leq E \tau^{\frac{5}{2}}\left(\int_{t_{n}}^{t_{n+1}}\left\|\nabla \mathbf{u}_{t t}\right\|_{0}^{2} d t\right)^{\frac{1}{2}}\left\|\nabla \varepsilon_{h}^{n+\frac{1}{2}}\right\|_{0} \\
& \leq \frac{E}{16}\left\|\nabla \varepsilon_{h}^{n+\frac{1}{2}}\right\|_{0}^{2} \tau+c E \tau^{4} \int_{t_{n}}^{t_{n+1}}\left\|\nabla \mathbf{u}_{t t}\right\|_{0}^{2} d t \\
(\mathrm{I})_{6} & \leq c \tau\left\|\nabla\left(\mathbf{u}^{n+\frac{1}{2}}-I_{h} \mathbf{u}^{n+\frac{1}{2}}\right)\right\|_{0}\left\|\nabla \varepsilon_{h}^{n+\frac{1}{2}}\right\|_{0} \\
& \leq \frac{E}{16}\left\|\nabla \varepsilon_{h}^{n+\frac{1}{2}}\right\|_{0}^{2} \tau+c E^{-1} \tau\left\|\nabla\left(\mathbf{u}^{n+\frac{1}{2}}-I_{h} \mathbf{u}^{n+\frac{1}{2}}\right)\right\|_{0}^{2}, \\
(\mathrm{I})_{7} & \leq c \tau h^{2}\left\|\nabla \varepsilon_{h}^{n+\frac{1}{2}}\right\|_{0}\left\|\bar{p}^{n+\frac{1}{2}}\right\|_{2} \leq \frac{E}{16}\left\|\nabla \varepsilon_{h}^{n+\frac{1}{2}}\right\|_{0}^{2} \tau+c E^{-1} h^{4} \int_{t_{n}}^{t_{n+1}}\|p\|_{2}^{2} d t
\end{aligned}
$$

For $(\mathrm{I})_{8}$ to $(\mathrm{I})_{12}$ in $(45)$ we can estimate using the definition of the trilinear function $d(\cdot, \cdot, \cdot)$ and its estimates:

$$
\begin{aligned}
(\mathrm{I})_{8} & \leq c \tau^{\frac{5}{2}}\left(\int_{t_{n}}^{t_{n+1}}\left[\left\|\mathbf{u}_{t t}\right\|_{0}^{2}\|\mathbf{u}\|_{2}^{2}+\left\|\mathbf{u}_{t}\right\|_{2}^{2}\left\|\mathbf{u}_{t}\right\|_{0}^{2}\right] d t\right)^{\frac{1}{2}}\left\|\nabla \varepsilon_{h}^{n+\frac{1}{2}}\right\|_{0} \\
& \leq \frac{E}{16}\left\|\nabla \varepsilon_{h}^{n+\frac{1}{2}}\right\|_{0}^{2} \tau+c E^{-1} \tau^{4} \int_{t_{n-1}}^{t_{n}}\left[\left\|\mathbf{u}_{t t}\right\|_{0}^{2}\|\mathbf{u}\|_{2}^{2}+\left\|\mathbf{u}_{t}\right\|_{2}^{2}\left\|\mathbf{u}_{t}\right\|_{0}^{2}\right] d t \\
(\mathrm{I})_{9} & \leq c \tau\left\|\nabla \varepsilon_{h}^{n+\frac{1}{2}}\right\|_{0}\left(\int_{t_{n}}^{t_{n+1}}\left\|\nabla \mathbf{u}_{t}\right\|_{0} d t\right)^{2} \\
& \leq \frac{E}{16}\left\|\nabla \varepsilon_{h}^{n+\frac{1}{2}}\right\|_{0}^{2} \tau+c E^{-1} \tau^{4} \sup _{0 \leq t \leq T}\left\|\nabla \mathbf{u}_{t}(t)\right\|_{0}^{2} \int_{t_{n}}^{t_{n+1}}\left\|\nabla \mathbf{u}_{t}\right\|_{0}^{2} d t \\
(\mathrm{I})_{10} & \leq c \tau^{\frac{5}{2}}\left\|\nabla \varepsilon_{h}^{n+\frac{1}{2}}\right\|_{0}\left\|\mathbf{u}^{n+\frac{1}{2}}\right\|_{2}\left(\int_{t_{n-1}}^{t_{n+1}}\left\|\mathbf{u}_{t t}\right\|_{0}^{2} d t\right)^{\frac{1}{2}} \\
& \leq \frac{E}{16}\left\|\nabla \varepsilon_{h}^{n+\frac{1}{2}}\right\|_{0}^{2} \tau+c E^{-1} \tau^{4} \sup _{0 \leq t \leq T}\|\mathbf{u}(t)\|_{2}^{2} \int_{t_{n}}^{t_{n+1}}\left\|\mathbf{u}_{t t}\right\|_{0}^{2} d t
\end{aligned}
$$




$$
\begin{aligned}
(\mathrm{I})_{11} \leq & c \tau\left\|\nabla \varepsilon_{h}^{n+\frac{1}{2}}\right\|_{0}\left(\left\|\nabla\left(T_{n}(\mathbf{u})-I_{h} T_{n}(\mathbf{u})\right)\right\|_{0}\left\|\nabla \mathbf{u}^{n+\frac{1}{2}}\right\|_{0}+\left\|T_{n}\left(\varepsilon_{h}\right)\right\|_{0}\left\|\mathbf{u}^{n+\frac{1}{2}}\right\|_{2}\right) \\
\leq & \frac{E}{16}\left\|\nabla \varepsilon_{h}^{n+\frac{1}{2}}\right\|_{0}^{2} \tau+c E^{-1} \tau\left\|\nabla\left(T_{n}(\mathbf{u})-I_{h} T_{n}(\mathbf{u})\right)\right\|_{0}^{2}\left\|\nabla \mathbf{u}^{n+\frac{1}{2}}\right\|_{0}^{2} \\
& +c E^{-1} \tau\left\|T_{n}\left(\varepsilon_{h}\right)\right\|_{0}^{2}\left\|\mathbf{u}^{n+\frac{1}{2}}\right\|_{2}^{2} \\
(\mathrm{I})_{12} \leq & c \tau\left(h^{-1}\left\|T_{n}\left(\varepsilon_{h}\right)\right\|_{0}+\left\|\nabla T_{n}\left(I_{h} \mathbf{u}\right)\right\|_{0}\right)\left\|\nabla\left(\mathbf{u}^{n+\frac{1}{2}}-I_{h} \mathbf{u}^{n+\frac{1}{2}}\right)\right\|_{0}\left\|\nabla \varepsilon_{h}^{n+\frac{1}{2}}\right\|_{0} \\
\leq & \frac{E}{16}\left\|\nabla \varepsilon_{h}^{n+\frac{1}{2}}\right\|_{0}^{2} \tau+c E^{-1} \tau\left\|T_{n}\left(\varepsilon_{h}\right)\right\|_{0}^{2}\left\|\mathbf{u}^{n+\frac{1}{2}}\right\|_{2}^{2} \\
& +c E^{-1} \tau\left\|\nabla T_{n}(\mathbf{u})\right\|_{0}^{2}\left\|\nabla\left(\mathbf{u}^{n+\frac{1}{2}}-I_{h} \mathbf{u}^{n+\frac{1}{2}}\right)\right\|_{0}^{2} .
\end{aligned}
$$

Summing up (45) from $n=1$ to $n=m-1$ and using the previous estimates for $(\mathrm{I})_{1}$ to $(\mathrm{I})_{12}$ and the regularities (A3) we obtain

$$
\begin{aligned}
\left\|\varepsilon_{h}^{m}\right\|_{0}^{2} & +E \tau \sum_{n=1}^{m}\left\|\nabla \varepsilon_{h}^{n}\right\|_{0}^{2} \\
& \leq C\left(\tau^{4}+h^{4}\right) \int_{0}^{T}\left[\left\|\mathbf{f}_{t t}\right\|_{0}^{2}+\|\mathbf{u}\|_{0}^{2}+\|p\|_{2}^{2}+\left\|\mathbf{u}_{t}\right\|_{2}^{2}+\left\|\nabla \mathbf{u}_{t t}\right\|_{0}^{2}\right] d t \\
& +C \tau \sum_{n=1}^{M-1}\left[\left\|\nabla\left(T_{n}(\mathbf{u})-I_{h} T_{n}(\mathbf{u})\right)\right\|_{0}^{2}+\left\|\nabla\left(\mathbf{u}^{n+\frac{1}{2}}-I_{h} \mathbf{u}^{n+\frac{1}{2}}\right)\right\|_{0}^{2}\right] \\
& +\tau \sum_{n=1}^{m-1} d_{n}\left\|\varepsilon_{h}^{n}\right\|_{0}^{2}
\end{aligned}
$$

where we write $d_{M-1}=c E^{-1}\left(\left\|\mathbf{u}\left(t_{M}\right)\right\|_{2}^{2}+\left\|\mathbf{u}\left(t_{M-1}\right)\right\|_{2}^{2}\right)$, and

$$
d_{n}=c E^{-1}\left(\left\|\mathbf{u}\left(t_{n+2}\right)\right\|_{2}^{2}+\left\|\mathbf{u}\left(t_{n+1}\right)\right\|_{2}^{2}+\left\|\mathbf{u}\left(t_{n}\right)\right\|_{2}^{2}\right)
$$

for $n=1, \cdots, M-2$. Now applying the discrete Gronwall inequality to (46) and using the regularities (A3), we deduce

$$
\begin{aligned}
\left\|\varepsilon_{h}^{m}\right\|_{0}^{2}+E \tau \sum_{n=1}^{m}\left\|\nabla \varepsilon_{h}^{n}\right\|_{0}^{2} \\
\leq \quad \exp \left\{\tau \sum_{n=1}^{m-1} d_{n}\right\}\left\{C\left(\tau^{4}+h^{4}\right)\right. \\
\quad \times \int_{0}^{T}\left[\left\|\mathbf{f}_{t t}\right\|_{0}^{2}+\|\mathbf{u}\|_{0}^{2}+\|p\|_{2}^{2}+\left\|\mathbf{u}_{t}\right\|_{2}^{2}+\left\|\nabla \mathbf{u}_{t t}\right\|_{0}^{2}\right] d t \\
\left.\quad+C \tau \sum_{n=1}^{M}\left[\left\|\nabla\left(T_{n}(\mathbf{u})-I_{h} T_{n}(\mathbf{u})\right)\right\|_{0}^{2}+\left\|\nabla\left(\mathbf{u}^{n+\frac{1}{2}}-I_{h} \mathbf{u}^{n+\frac{1}{2}}\right)\right\|_{0}^{2}\right]\right\} \\
\leq \quad C\left(\tau^{4}+h^{4}\right) \int_{0}^{T}\left[\left\|\mathbf{f}_{t t}\right\|_{0}^{2}+\|\mathbf{u}\|_{0}^{2}+\|p\|_{2}^{2}+\left\|\mathbf{u}_{t}\right\|_{2}^{2}+\left\|\nabla \mathbf{u}_{t t}\right\|_{0}^{2}\right] d t \\
\quad+C \tau \sum_{n=1}^{M}\left[\left\|\nabla\left(T_{n}(\mathbf{u})-I_{h} T_{n}(\mathbf{u})\right)\right\|_{0}^{2}+\left\|\nabla\left(\mathbf{u}^{n+\frac{1}{2}}-I_{h} \mathbf{u}^{n+\frac{1}{2}}\right)\right\|_{0}^{2}\right] .
\end{aligned}
$$


But the last two terms in (47) can be estimated by using the approximation property (22) of projection $\mathrm{I}_{h}$ as follows:

$$
\begin{aligned}
& \tau\left\|\nabla\left(\mathbf{u}^{n+\frac{1}{2}}-I_{h} \mathbf{u}^{n+\frac{1}{2}}\right)\right\|_{0}^{2} \\
\leq & 3 \tau\left\{\left\|\nabla\left(\mathbf{u}^{n+\frac{1}{2}}-\overline{\mathbf{u}}^{n+\frac{1}{2}}\right)\right\|_{0}^{2}+\left\|\nabla\left(\overline{\mathbf{u}}^{n+\frac{1}{2}}-I_{h} \overline{\mathbf{u}}^{n+\frac{1}{2}}\right)\right\|_{0}^{2}+\left\|\nabla\left(I_{h} \overline{\mathbf{u}}^{n+\frac{1}{2}}-I_{h} \mathbf{u}^{n+\frac{1}{2}}\right)\right\|_{0}^{2}\right\} \\
\leq & c\left(\tau^{4}+h^{4}\right) \int_{t_{n}}^{t_{n+1}}\left[\left\|\nabla \mathbf{u}_{t t}\right\|_{0}^{2}+\|\mathbf{u}\|_{3}^{2}\right] d t \\
& \tau\left\|\nabla\left(T_{n}(\mathbf{u})-I_{h} T_{n}(\mathbf{u})\right)\right\|_{0}^{2} \\
= & \tau\left\|\nabla\left[\left(\mathbf{u}^{n+\frac{1}{2}}-\mathbf{u}^{n+\frac{1}{2}}-T_{n}(\mathbf{u})\right)-I_{h}\left(\mathbf{u}^{n+\frac{1}{2}}-\mathbf{u}^{n+\frac{1}{2}}-T_{n}(\mathbf{u})\right)\right)\right\|_{0}^{2} \\
\leq & \left.3 \tau \| \nabla \mathbf{u}^{n+\frac{1}{2}}-I_{h} \mathbf{u}^{n+\frac{1}{2}}\right)\left\|_{0}^{2}+3 \tau\right\| \nabla \int_{t_{n}}^{t_{n+1}}\left(\mathbf{u}_{t}-I_{h} \mathbf{u}_{t}\right) d t \|_{0}^{2} \\
+ & 3 \tau\left\|\nabla \int_{t_{n-1}}^{t_{n}}\left(\mathbf{u}_{t}-I_{h} \mathbf{u}_{t}\right) d t\right\|_{0}^{2} \\
\leq & c\left(\tau^{4}+h^{4}\right) \int_{t_{n}}^{t_{n+1}}\left[\left\|\nabla \mathbf{u}_{t t}\right\|_{0}^{2}+\left\|\mathbf{u}_{t}\right\|_{2}^{2}+\|\mathbf{u}\|_{3}^{2}\right] d t .
\end{aligned}
$$

Now the desired estimate (42) follows from (31) by the triangle inequality, (47)-(49), and the projection approximation $(22)$. $\sharp$

\section{$5 \quad$ Numerical simulations}

\subsection{Triaxial ellipsoidal tetrahedral mesh}

The essential strategy for generating a tetrahedral mesh suitable for a triaxial ellipsoidal cavity is first to construct a spherical tetrahedral mesh [6] which is then deformed into a triaxial ellipsoidal geometry by introducing the eccentricity $\mathcal{E}$ as a geometric parameter of the triaxial ellipsoidal mesh. More precisely, all nodes $\left(x_{i}, y_{i}, z_{i}\right)$ in a spherical tetrahedral mesh within the unit sphere satisfying

$$
x_{i}^{2}+y_{i}^{2}+z_{i}^{2}=r_{i}^{2}, \quad 0<r_{i} \leq 1,
$$

can be transformed by

$$
x_{i}^{\mathcal{E}}=x_{i}, \quad y_{i}^{\mathcal{E}}=y_{i} \sqrt{1+\mathcal{E}^{2}}, \quad z_{i}^{\mathcal{E}}=z_{i} \sqrt{1-\mathcal{E}^{2}}
$$

such that the deformed nodes $\left(x_{i}^{\mathcal{E}}, y_{i}^{\mathcal{E}}, z_{i}^{\mathcal{E}}\right)$ satisfy

$$
\left(x_{i}^{\mathcal{E}}\right)^{2}+\frac{\left(y_{i}^{\mathcal{E}}\right)^{2}}{1+\mathcal{E}^{2}}+\frac{\left(z_{i}^{\mathcal{E}}\right)^{2}}{1-\mathcal{E}^{2}}=r_{i}^{2}, \quad 0<r_{i} \leq 1 .
$$

For the purpose of resolving the thin viscous boundary layer, we can construct more nodes in the vicinity of the bounding surface of the triaxial ellipsoidal cavity by stretching the spherical mesh points $\left(x_{i}, y_{i}, z_{i}\right)$ radially before the deformation, for example,

$$
\left[\begin{array}{l}
x_{i} \\
y_{i} \\
z_{i}
\end{array}\right]=\frac{1}{r_{i}} \sin \left(\frac{\pi}{2} r_{i}\right)^{2 / 3}\left[\begin{array}{l}
x_{i} \\
y_{i} \\
z_{i}
\end{array}\right] .
$$




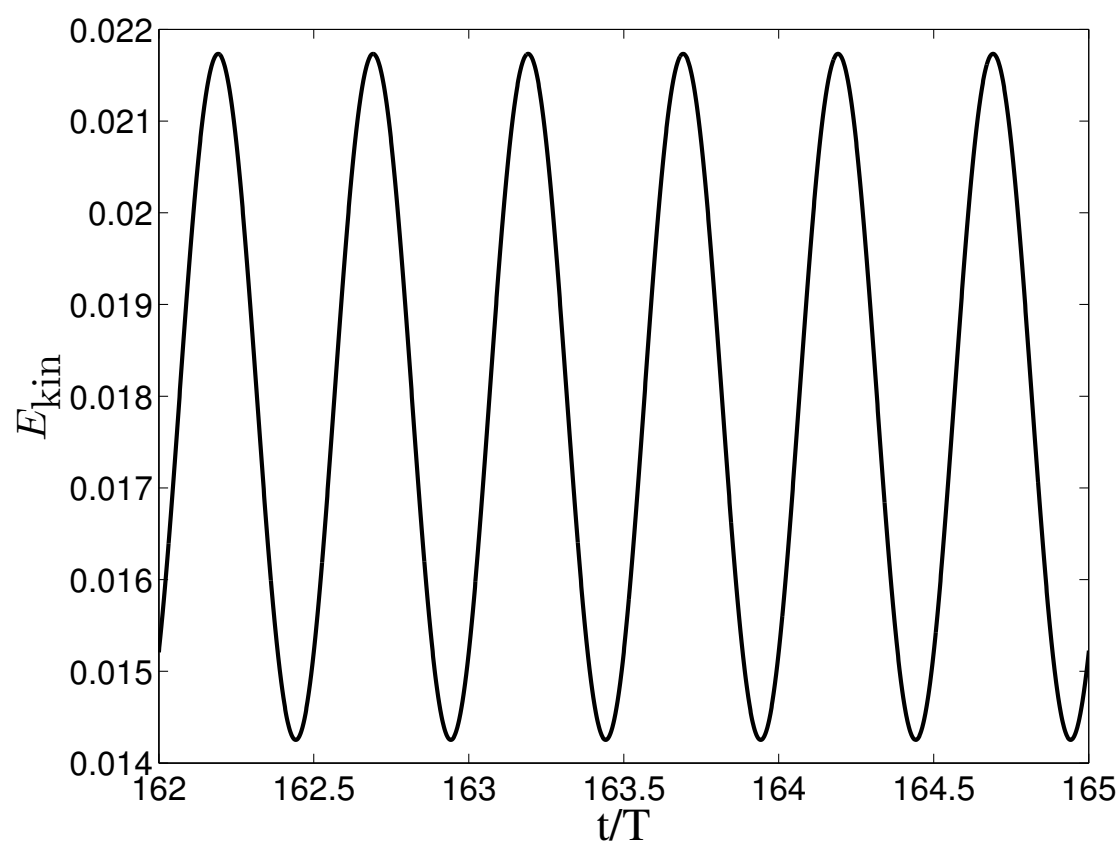

Figure 1: Kinetic energy, $E_{\text {kin }}(t)$, obtained with the implicit scheme is shown as a function of the scaled time $(T=2 \pi / \hat{\omega})$ at a fixed $\mathcal{E}=0.5$ for $E=10^{-4}, \hat{\omega}=1.2$ and $P o=0.3$.

The spherical mesh itself begins with approximating the sphere by an icosahedron which is then further divided into 20 identical tetrahedra based on its 20 triangular facets and the center of the sphere. This initial tetrahedral mesh is then refined recursively by subdividing each of the tetrahedra into eight subtetrahedra. The three-dimensional tetrahedralization of the triaxial ellipsoid produces a finite element mesh that does not have pole or central numerical singularities. When $\mathcal{E}$ is very close to 1 , representing a highly flatted triaxial ellipsoidal disk, an alternative meshing algorithm should be used. This is because a regular shaped tetrahedron after transformation may become too stretched and, consequently, lead to a poor finite element approximation. In this case, a general mesh generation algorithm based on the Delaunay triangulation can be employed instead.

\subsection{Implementation and results}

In the previous studies [2, 27], an explicit Crank-Nicolson scheme was employed for numerical simulation, which may result in numerical instabilities in the strongly nonlinear regime and, thus, limit the size of time steps in numerical integration. On the basis of the present study, we implement the second-order implicit Crank-Nicolson scheme, defined by the finite element system (39) and (40), in a new finite element code for triaxial ellipsoidal geometry.

After implementation, we have simulated a number of nonlinear solutions using the new implicit code in a triaxial ellipsoidal cavity with $\mathcal{E}=0.5$. Figure 1 shows the time-dependent kinetic energies, $E_{\text {kin }}(t)$, defined as

$$
E_{\text {kin }}(t)=\frac{1}{2 \Omega} \int_{\Omega}|\mathbf{u}(\mathbf{r}, t)|^{2} \mathrm{~d} \Omega
$$



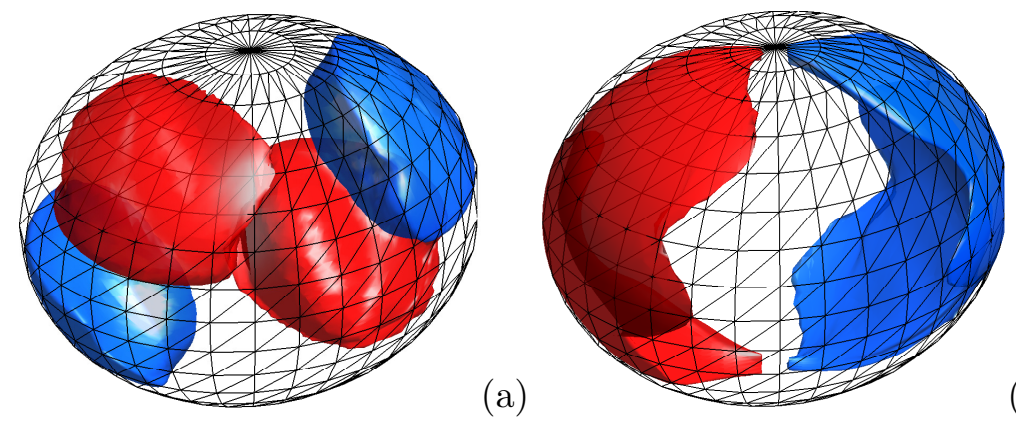

(a)

Figure 2: (a) Isosurface of the radial component of the flow and (b) isosurface of the latitudinal component for $\mathcal{E}=0.5, E=10^{-4}, \hat{\omega}=1.2$ and $P o=0.3$.

for a nonlinear librating flow as a function of time for $P o=0.3$ and $E=10^{-4}$, where $\int_{\Omega}$ denotes the integral over the triaxial ellipsoidal cavity. The corresponding spatial structure of the flow is depicted in Figure 2. It reveals that the numerical solutions obtained with the implicit scheme are consistent with both the analytical solution [27] and the numerical solution based on the explicit scheme [2]. However, the new numerical code using the implicit Crank-Nicolson scheme is much more efficient, being numerically stable with larger time steps. Moreover, since the nonlinear effect taking place in the Ekman boundary layer plays an essential role in generating the mean flow, the implicit Crank-Nicolson scheme would help capture the boundary-layer nonlinear effect more accurately.

\section{Concluding remarks}

As a result of rapid rotation and interaction between planets and stars, many planetary bodies are in the shape of a triaxial ellipsoid. This paper presents the theoretical analysis for a finite element method that can be used to compute nonlinear time-dependent librating flows confined in librating triaxial ellipsoidal cavities with arbitrary eccentricity $0 \leq \mathcal{E}<1$, providing a mathematical foundation for the geophysical and astrophysical application of the numerical method. It can be readily extended to other problems of geophysical and astrophysical fluid dynamics, such as tidally or precessionally driven flow, in non-spherical geometry.

In comparison to the spectral method, the finite element method is based on the threedimensional triangulation of a triaxial ellipsoidal domain with the velocity and pressure being represented by continuous piecewise quadratic and linear finite elements. We have discussed the stability properties of the finite element solution and estimated the numerical errors of the finite element approximation. We have also implemented the second-order implicit scheme which is then used to simulate several nonlinear flows a triaxial ellipsoid. To authors' best knowledge, this paper represents the first theoretical study on a finite element scheme for simulating a nonlinear librating flow in triaxial ellipsoidal geometry.

The numerical scheme presented in this paper would be also suitable for simulating dynamo action taking place in nearly synchronous planets and moons that are thermally or chemically non-convective. Although it is widely accepted that thermal or chemical buoyancy within planetary fluid cores drives planetary dynamo, exceptional cases may exist for certain planets, such as Mercury and Ganymede, which may require an alternative mechanism of sustaining their 
dynamo action. An extension of a similar theoretical study to include both the magnetic field on the flow and the dynamo action in a librating triaxial ellipsoid would be challenging.

\section{Acknowledgment}

KHC was supported by Hong Kong RGC grant (Project 700310), YH was supported by the NSF of China grant 10971166, KZ was supported by U.K. NERC, Royal Society, Leverhulme grants and JZ was supported by Hong Kong RGC grant (Project 405110). Part of the work was carried out when $\mathrm{YH}$ and $\mathrm{KZ}$ were visiting respectively Department of Mathematics and Institute of Mathematical Sciences, the Chinese University of Hong Kong and supported by a Direct Grant for Research from CUHK.

\section{References}

[1] K. Chan, K. Zhang, J. Zou, Spherical interface dynamos: mathematical theory, finite element approximation, and application, SIAM J. Numer. Anal. 44 (2006) 1877-1902.

[2] K. Chan, X. Liao, K. Zhang, Simulations of fluid motion in spheroidal planetary cores driven by latitudinal libration, Physics of the Earth and Planetary Interiors 187 (2011) 404-415.

[3] D. Cebron, M. Le Bars, J. Leontini, P. Maubert, P. Le Gal, A systematic numerical study of the tidal instability in a rotating triaxial ellipsoid, Physics of the Earth and Planetary Interiors 182 (2010) 119-128.

[4] Z. Chen, J. Zou, Finite element methods and their convergence for elliptic and parabolic interface problems, Numerische Mathematik 79 (1998) 175-202.

[5] P.G. Ciarlet, The Finite Element Method for Elliptic Problems, first ed., North-Holland Pub. Co., Amsterdam/New York, 1978.

[6] M.E. Everett, A three-dimensional spherical mesh generator, Geophysical Journal International 130 (1997) 193-200.

[7] M. Feistauer, A. Zenisek, Finite element solution of nonlinear elliptic problems, Numerische Mathematik 50 (1987) 451-475.

[8] V. Girault, P. Raviart, Finite Element Approximation of the Navier-Stokes Equations, Springer: New York, 1981.

[9] V. Girault, P. Raviart, Finite Element Methods for Navier-Stokes Equations: Theory and Algorithms, Springer: Berlin, 1986.

[10] Y.N. He, Fully discrete stabilized finite element method for the time-dependent NavierStokes equations, IMA J. Numer. Anal. 23 (2003) 1-27.

[11] Y.N. He, Two-level methods based on finite element and Crank-Nicolson extrapolation for the time-dependent Navier-Stokes equations, SIAM J. Numer. Anal. 41 (2003) 1263-1285. 
[12] Y.N. He, W.W. Sun, Stabilized finite element methods based on Crank-Nicolson extrapolation scheme for the time-dependent Navier-Stokes equations, Math. Comp. 76 (2007) $115-136$.

[13] Y.N. He, The Euler implicit/explicit scheme for the 2D time-dependent Navier-Stokes equations with smooth or non-smooth initial data, Math. Comp. 77 (2008) 2097-2124.

[14] J.G. Heywood, R. Rannacher, Finite element approximation of the nonstationary NavierStokes problem I: Regularity of solutions and second-order error estimates for spatial discretization, SIAM J. Numer. Anal. 19 (1982) 275-311.

[15] J.G. Heywood, R. Rannacher, Finite element approximation of the nonstationary NavierStokes problem III: Smoothing property and high order error estimates for spatial discretization, SIAM J. Numer. Anal. 25 (1988) 489-512.

[16] J.G. Heywood, R. Rannacher, Finite element approximation of the nonstationary NavierStokes problem IV: Error Analysis for second-order time discretization, SIAM J. Numer. Anal. 27 (1990) 353-384.

[17] H. Johnston, J.G. Liu, Accurate, stable and efficient Navier-Stokes solvers based on explicit treatment of the pressure term, J. Computational Physics 199 (2004) 221-259.

[18] D. Kong, K. Zhang, G. Schubert, Shapes of two-layer models of rotating planets, Journal of Geophysical Research 115 (2010) E12003.

[19] S. Lorenzani, A. Tilgner, Fluid instabilities in precessing spheroidal cavities, Journal of Fluid Mechanics 447 (2001) 111-128.

[20] J.L. Margot, S.J. Peale, R.F. Jurgens, M.A. Slade, I.V. Holin, Large longitude libration of mercury reveals a molten core, Science 316 (2007) 710-714.

[21] M. Marion, R. Temam, Navier-Stokes equations: Theory and approximation, in: Handbook of Numerical Analysis, Vol. VI, 503-688, North-Holland, Amsterdam, 1998.

[22] H. Matsui and H. Okuda, Treatment of the magnetic field for geodynamo simulations using the finite element method, Earth Planets Space, 56 (2004), 945-954.

[23] D. Schmitt, Numerical study of viscous modes in a rotating spheroid, Journal of Fluid Mechanics 567 (2006) 399-414.

[24] J. Shen, Long time stability and convergence for fully discrete nonlinear Galerkin methods, Appl. Anal. 38 (1990) 201-229.

[25] F. Tone, Error analysis for a second order scheme for the Navier-Stokes equations, Applied Numerical Mathematics 50 (2004) 93-119.

[26] O.C. Zienkiewicz, The finite element method, third ed., McGraw-Hill: London, 1977.

[27] K. Zhang, K. Chan, X. Liao, Asymptotic theory of resonant flow in a spheroidal cavity driven by latitudinal libration, Journal of Fluid Mechanics 692 (2012) 420-445. 